\title{
Prostate volume reduction following pure transurethral bipolar plasma vaporization and conventional transurethral resection of the prostate: a prospective investigation using transrectal 3D ultrasound volumetry
}

Kranzbühler, Benedikt ; Gross, Oliver ; Fankhauser, Christian D ; Wettstein, Marian S ; Grossmann, Nico C ; Hefermehl, Lukas J ; Zimmermann, Matthias ; Müller, Alexander ; Eberli, Daniel ; Sulser, Tullio ; Poyet, Cédric ; Hermanns, Thomas

\begin{abstract}
PURPOSE To evaluate and compare postoperative changes in prostate volume and clinical outcome after bipolar plasma vaporization (BPV) and conventional transurethral resection of the prostate (TURP). PATIENTS AND METHODS Consecutive series of patients undergoing BPV or TURP were included in this prospective, nonrandomized study. Planimetric volumetry after transrectal threedimensional ultrasound of the prostate was performed preoperatively and postoperatively after 6 weeks, 6 months and 12 months. Additionally, changes in clinical outcome parameters were assessed and compared between the groups. The reduction ratio and analysis of covariance were used to compare volume changes between BPV and TURP. Multiple regression analysis was performed to assess a possible interaction between preoperative prostate volume and effect of therapy. RESULTS A total of 157 patients were included (BPV: $\mathrm{n}=68$, TURP: $\mathrm{n}=89$ ). Median preoperative prostate volume was $43.1 \mathrm{ml}$ in the BPV group and $45.9 \mathrm{ml}$ in the TURP group $(\mathrm{p}=0.43)$. Postoperatively, the prostate volumes decreased significantly in both groups. After catheter removal, the relative residual prostate volume was significantly higher in the BPV group (66.6 vs. $60.8 \%$; $=0.02)$. Thereafter, significant differences were not detectable anymore (12 months: 46.6 vs. $47.1 \%$ p $=0.82)$. Regression analysis revealed that tissue ablation after BPV was superior to TURP in prostates $<45 \mathrm{ml}$ but inferior in prostates $>45 \mathrm{ml}$. All clinical outcome parameters improved significantly and were not significantly different between the groups. CONCLUSIONS Volume reduction and short-term clinical outcome following pure BPV was excellent and comparable to conventional TURP. However, volume reduction seems to be limited in patients with larger prostates.
\end{abstract}

DOI: https://doi.org/10.1007/s00345-016-1876-5

Posted at the Zurich Open Repository and Archive, University of Zurich

ZORA URL: https://doi.org/10.5167/uzh-124746

Journal Article

Accepted Version

Originally published at:

Kranzbühler, Benedikt; Gross, Oliver; Fankhauser, Christian D; Wettstein, Marian S; Grossmann, Nico C; Hefermehl, Lukas J; Zimmermann, Matthias; Müller, Alexander; Eberli, Daniel; Sulser, Tullio; Poyet, Cédric; Hermanns, Thomas (2017). Prostate volume reduction following pure transurethral bipolar plasma vaporization and conventional transurethral resection of the prostate: a prospective investigation using transrectal 3D ultrasound volumetry. World Journal of Urology, 35(3):429-435. 
DOI: https://doi.org/10.1007/s00345-016-1876-5 


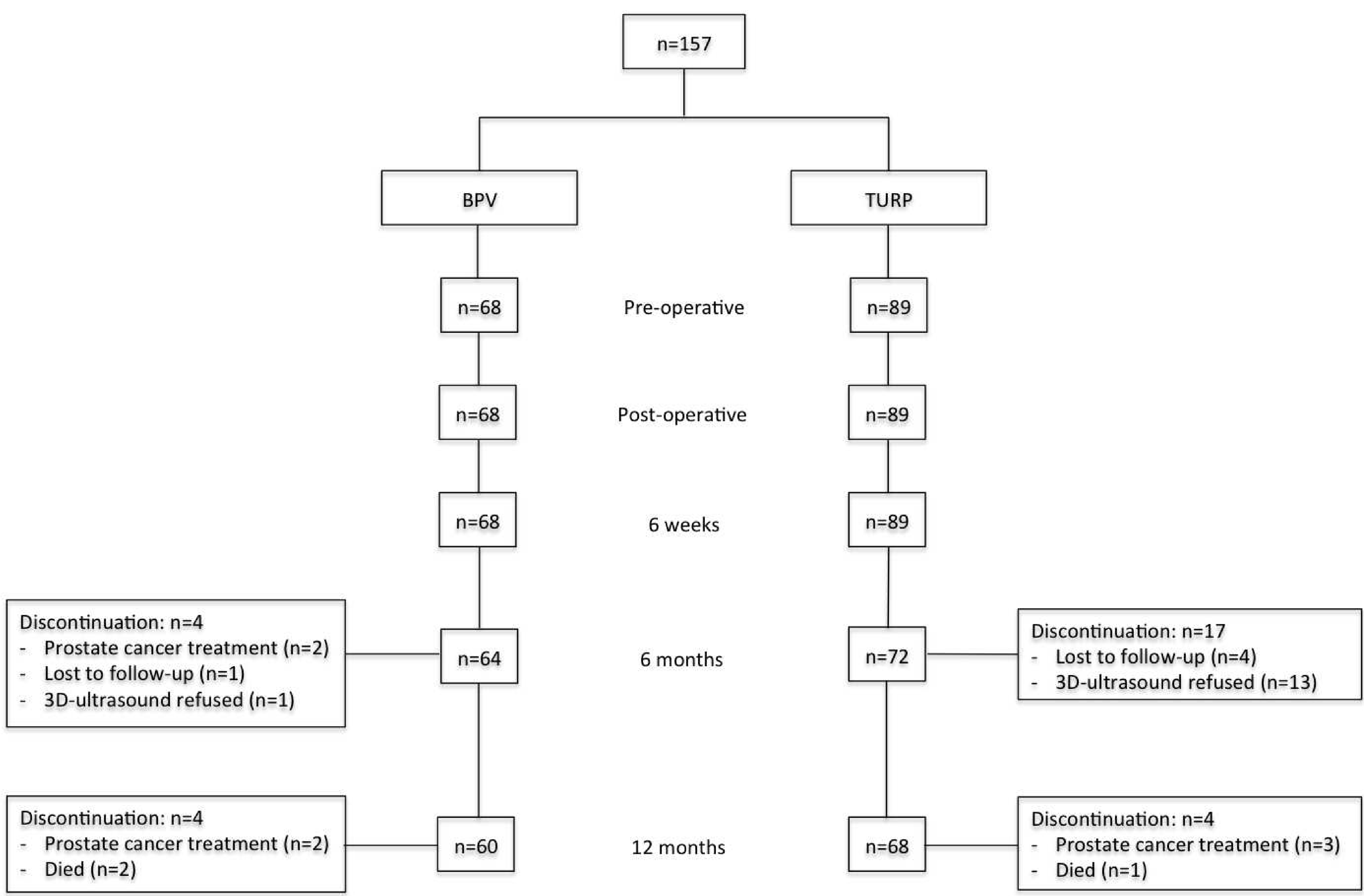




\section{after 12 months}

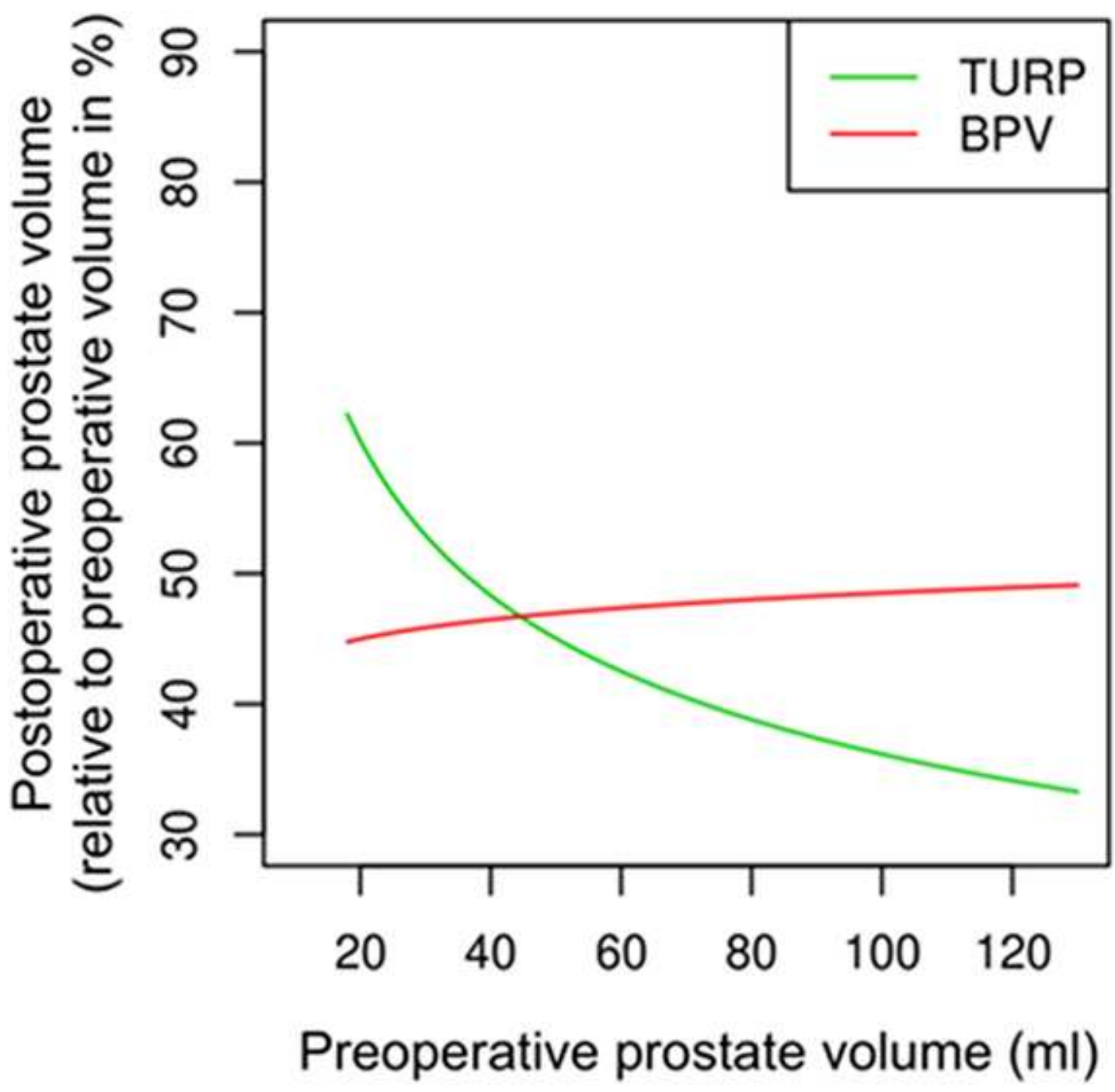


Table 1 - Baseline parameters of all 157 patients (a), Intra and perioperative outcome (b),

Postoperative outcome (c), Changes of the investigated follow-up parameters (d)

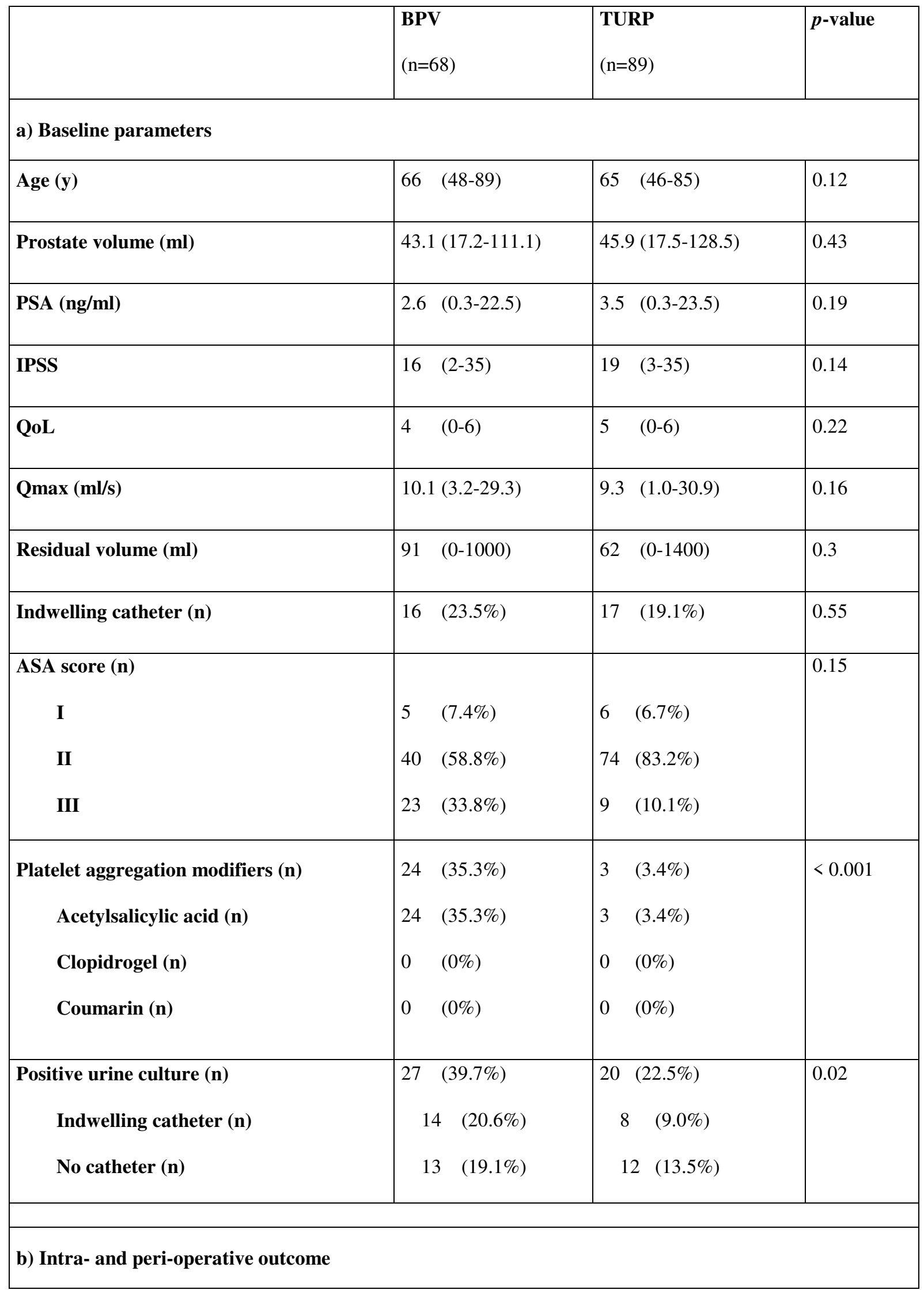




\begin{tabular}{|c|c|c|c|}
\hline Operative time (min) & $77.5(34-145)$ & $70(10-165)$ & 0.16 \\
\hline \multicolumn{4}{|l|}{ Anesthesia: } \\
\hline Intubation (n) & $54 \quad(79.4 \%)$ & $68(76.4 \%)$ & 0.7 \\
\hline Spinal anesthesia (n) & $14 \quad(20.6 \%)$ & $21(23.6 \%)$ & 0.7 \\
\hline Blood transfusion (n) & 0 & 0 & $\mathrm{n} / \mathrm{a}$ \\
\hline Duration of catheterization (d) & $3 \quad(2-13)$ & $3 \quad(2-22)$ & 0.65 \\
\hline Re-catheterization (n) & $9 \quad(13.3 \%)$ & $10(11.2 \%)$ & 0.81 \\
\hline Urinary retention $(\mathbf{n})$ & $7 \quad(10.3 \%)$ & $9(10.1 \%)$ & \\
\hline Residual volume $>300 \mathrm{ml}(\mathrm{n})$ & $1 \quad(1.5 \%)$ & $0 \quad(0 \%)$ & \\
\hline Persistent haematuria (n) & $1 \quad(1.5 \%)$ & $0 \quad(0 \%)$ & \\
\hline Stress urinary incontinence (n) & $0 \quad(0 \%)$ & $1 \quad(1.1 \%)$ & \\
\hline \multicolumn{4}{|l|}{ c) Post-operative outcome } \\
\hline Urinary tract infection (6 weeks; $n$ ) & $12(17.6 \%)$ & $2(2.2 \%)$ & 0.001 \\
\hline \multicolumn{4}{|l|}{ Dysuria (n) } \\
\hline 6 weeks & $26(38.2 \%)$ & $28(31.5 \%)$ & 0.4 \\
\hline 6 months & $2 \quad(2.9 \%)$ & $2(2.2 \%)$ & 0.99 \\
\hline 12 months & $3 \quad(4.4 \%)$ & $3(3.4 \%)$ & 0.99 \\
\hline Urethral stricture (n) & $3 \quad(4.4 \%)$ & $3(3.4 \%)$ & 0.99 \\
\hline Re-operation (n) & $\begin{array}{ll}0 & (0 \%)\end{array}$ & $0 \quad(0 \%)$ & $\mathrm{n} / \mathrm{a}$ \\
\hline \multicolumn{4}{|c|}{ d) Changes of the investigated follow-up parameters } \\
\hline \multicolumn{4}{|l|}{ IPSS } \\
\hline Preoperative & $16 \quad(2-35)$ & $19 \quad(3-35)$ & 0.14 \\
\hline Postoperative & $\mathrm{n} / \mathrm{a}$ & $\mathrm{n} / \mathrm{a}$ & \\
\hline 6 weeks & $8 \quad(0-29)$ & $8 \quad(0-33)$ & 0.58 \\
\hline 6 months & $3 \quad(0-24)$ & $4 \quad(0-25)$ & 0.84 \\
\hline
\end{tabular}




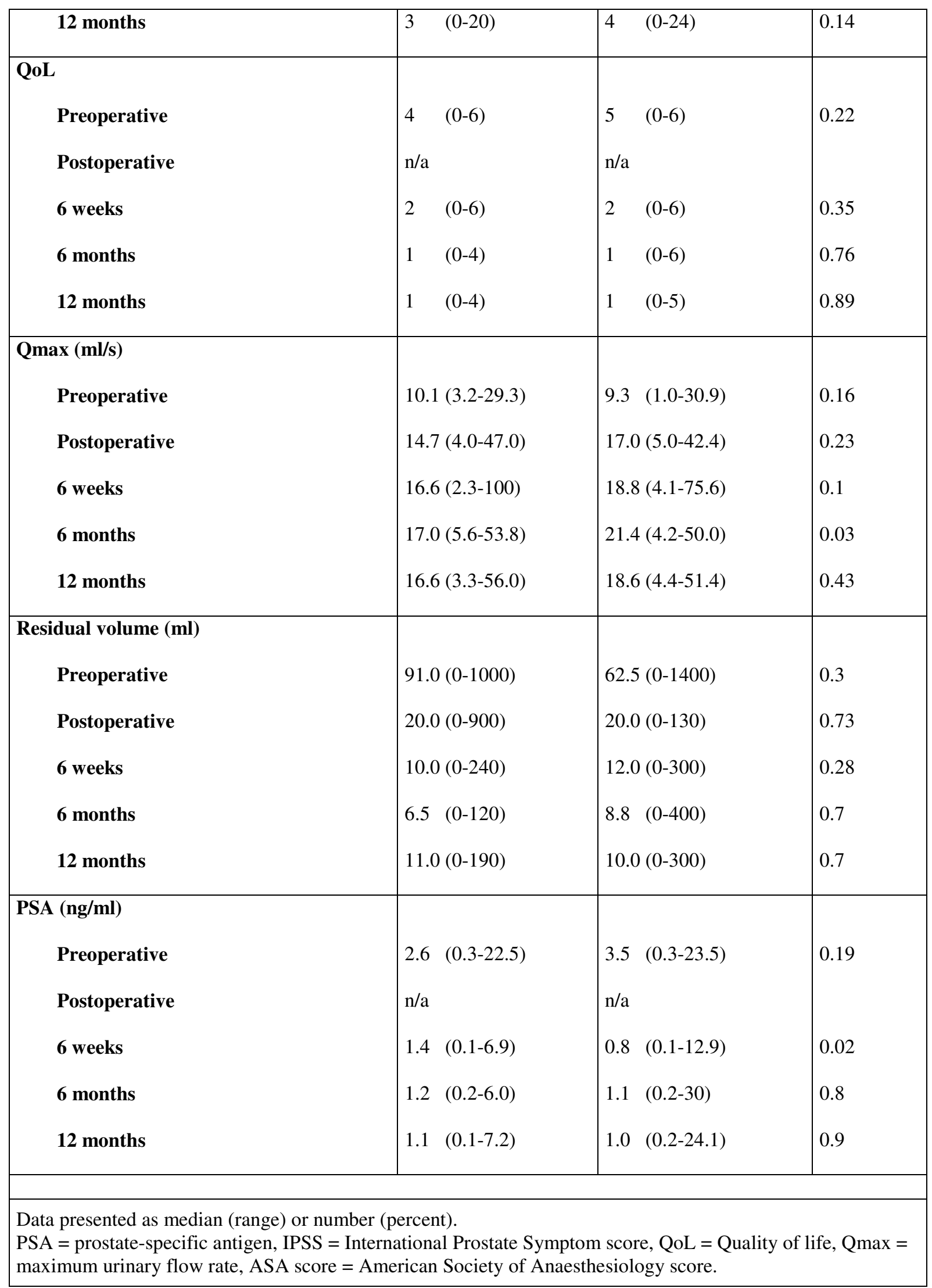


Table 2 - Post-operative prostate volumes in relation to pre-operative prostate volumes

\begin{tabular}{|c|c|c|c|c|}
\hline & \multicolumn{2}{|c|}{ Prostate volume } & \multicolumn{2}{|c|}{ Volume reduction ratio } \\
\hline & BPV & TURP & Raw ratio $^{\S}$ & $\begin{array}{c}\text { Adjusted ratio }^{\circ} \\
\text { (ANCOVA) }\end{array}$ \\
\hline Pre-operative & $43.1(17.2-111.1)$ & $45.9(17.5-128.5)$ & & \\
\hline \multicolumn{5}{|l|}{ Post-operative } \\
\hline & $26.8 \mathrm{ml}(10.3-89.0)^{\mathrm{a}}$ & $27.6 \mathrm{ml}(11.2-90.1)^{\mathrm{a}}$ & $1.10(1.02-1.18)$ & $1.08(1.01-1.16)$ \\
\hline & $66.6 \%$ & $60.8 \%^{\mathrm{b}}$ & $p=0.019$ & $p=0.032$ \\
\hline \multicolumn{5}{|l|}{ Six weeks } \\
\hline & $21.1 \mathrm{ml}(7.0-68.6)^{\mathrm{a}}$ & $24.4 \mathrm{ml}(11.3-92.7)^{\mathrm{a}}$ & $0.98(0.90-1.06)$ & $0.97(0.89-1.05)$ \\
\hline & $52.4 \%^{\mathrm{b}}$ & $53.7 \%{ }^{\mathrm{b}}$ & $p=0.57$ & $p=0.42$ \\
\hline \multicolumn{5}{|l|}{ Six months } \\
\hline & $19.2 \mathrm{ml}(5.5-59.2)^{\mathrm{a}}$ & $20.6 \mathrm{ml}(10.1-87.9)^{\mathrm{a}}$ & $0.98(0.90-1.08)$ & $0.98(0.90-1.07)$ \\
\hline & $48.2 \%{ }^{\mathrm{b}}$ & $49.0 \%$ & $p=0.72$ & $p=0.68$ \\
\hline \multicolumn{5}{|l|}{ Twelve months } \\
\hline & $18.3 \mathrm{ml}(5.0-59.9)^{\mathrm{a}}$ & $21.0 \mathrm{ml}(8.0-89.0)^{\mathrm{a}}$ & $0.99(0.89-1.10)$ & $0.98(0.89-1.08)$ \\
\hline & $46.6 \%{ }^{\mathrm{b}}$ & $47.1 \%{ }^{\mathrm{b}}$ & $p=0.82$ & $p=0.71$ \\
\hline \multicolumn{5}{|c|}{$\begin{array}{l}{ }^{\mathrm{a}} \text { Median prostate prostate volume (range) } \\
{ }^{\mathrm{b}} \text { Residual prostate volume relative to pre-operative prostate volume in percent } \\
{ }^{\S} \text { Change score in log prostate volume relative to log baseline prostate volume (95\%-confidence interval); } \\
\text { corresponding } p \text {-value (t-Test). } \\
{ }^{\circ} \text { Analysis of covariance (ANCOVA) with adjustment for pre-operative prostate volume (95\%-confidence } \\
\text { interval); corresponding } p \text {-value. }\end{array}$} \\
\hline
\end{tabular}

